\title{
Una quarantena può durare anche "solo" quattordici giorni
}

\section{Lorenzo Tomasin}

PUBBLICATO: 20 MARZO 2020

\section{Quesito:}

In occasione della drammatica diffusione del coronavirus, sono pervenute in redazione alcune domande che chiedono se sia lecito usare la parola quarantena, che si riferisce a un periodo di quaranta giorni, per il periodo di isolamento di quattordici giorni a cui sono sottoposti coloro che sono stati in contatto con i malati o che comunque sono sospetti di essere stati contagiati.

\section{Una quarantena può durare anche "solo" quattordici giorni}

$\mathrm{L}$

a parola quarantena è formata a partire da quaranta con il suffisso -ena, che in varie lingue romanze è impiegato per i numerali ordinali, e che a sua volta rimonta alla terminazione applicata in latino ai distributivi del tipo di novēni 'nove per volta', dèni 'dieci per volta', vicēni 'venti per volta'.

In età medievale, questa parola indicava - in italiano come nelle altre lingue romanze - un periodo di quaranta giorni con riferimento a pratiche devozionali, liturgiche o penitenziali: si faceva una quarantena come si fa ancora oggi una novena di preghiera o simili; oppure si lucrava una quarantena (cioè quaranta giorni di "sconto") nelle pratiche delle indulgenze. Di fatto, fino al secolo XVI non sembra sia attestato in italiano il significato oggi corrente di questa parola.

Ancora la prima impressione del Vocabolario degli Accademici della Crusca (I6r2), basata come è noto soprattutto sullo spoglio di testi medievali toscani, mette a lemma la forma quarantina, ma la spiega con: "quarantena, numero di quaranta, come decina, dodicina, o dozzina, e s'applica a pena, o a indulgenzia, che più comunemente diciamo quarantena".

L'impiego del termine quarantena nel significato di 'periodo di isolamento sanitario' (che in molti dialetti italiani non si riferisce, peraltro, solo ai sospetti appestati, ma anche alle puerpere messe a riposo dopo il parto) non sembra essersi diffuso prima del Cinquecento.

In particolare, la parola quarantena nell'accezione odierna è impiegata forse per la prima volta a Milano alla fine del XVI secolo. Qui, una quarantena, insieme devozionale e sanitaria, è imposta dalle autorità civili (spagnole) e religiose nell'autunno del 1576 per una durata canonica di quaranta giorni, in occasione di una pestilenza rimasta poi celebre (la cosiddetta Peste di San Carlo). Dopo la fine di quella quarantena, nel 1577 si prospetta la possibilità di "ridurre di nuovo la detta città a una quarantena, almeno per quindeci giorni”, come recita una grida del marzo I577. Già a quest'altezza cronologica, dunque, il termine quarantena slitta rapidamente dal significato originario di "periodo di quaranta giorni' a quello traslato di 'periodo di applicazione di misure sanitarie', indipendentemente dalla loro durata.

L'impiego di quarantena con la specificazione della sua estensione, anche diversa da quella delle antiche quarantene religiose, è comune nei testi medici e in quelli giuridici italiani dei secoli seguenti. In un trattato Del governo della peste pubblicato nel i7ı4, Ludovico Antonio Muratori dedica un intero capitolo a "Luogo e regole della quarantena", soffermandosi anche sulla sua durata: "il tempo della 
quarantena - scrive Muratori - secondo la pratica de' prudenti maestrati di Venezia, ora è di pochi, ora è di molti giorni, prendendosi la misura di ciò dal maggiore o minor pericolo, e sospetto, e dalla maggiore o minor lontananza dell'infezione. Lintera quarantena è di 40 dì, dal che venne il suo nome, e tanto si suol richiedere negli urgenti sospetti di Peste". Ma per altre circostanze, continua il grande erudito modenese, "mi dà animo di francamente asserire essere bastevoli 20 giorni di quarantena".

È chiaro, dunque, che nell'uso della trattatistica italiana la parola quarantena è impiegata da vari secoli anche per periodi di durata diversa da quaranta giorni. L'uso diviene assolutamente normale sia nei testi medici, sia in quelli giuridici del secolo XIX: così è ad esempio nel Regolamento sulle Quarantene e Sciorini decretato dal Magistrato di Sanità sedente in Genova il I2 maggio I8I7 (dove si danno le diverse durate delle quarantene previste per i vascelli), o ancora nel Dizionario di igiene pubblica e di polizia sanitaria ad uso dei medici e dei magistrati di Francesco Freschi, pubblicato a Torino nel i86o. L'uso è condiviso anche da illustri scrittori otto-novecenteschi, come Tommaseo ("trentacinque giorni di quarantena") e Rebora ("smonto per 5 giorni di quarantena": questo e il precedente esempio vengono dal GDLI).

Nell'accezione sanitaria che ci interessa, il termine quarantena si è diffuso - verosimilmente a partire dall'italiano, nel corso del secolo XVII - in tutte le principali lingue europee, comprese quelle (come l'inglese, quarantine, o il tedesco, Quarantäne) nelle quali il legame con la parola che significa quaranta è ovviamente venuto meno.

Non c'è dunque alcuna necessità di coniare una parola nuova per indicare il periodo di quattordici giorni di isolamento attualmente adottato per limitare la diffusione del Covid-Ig. Peraltro, l'italiano già dispone di almeno due sinonimi di quarantena che sono stati usati in passato con riferimento a periodi diversi da quaranta giorni: spurgazione e contumacia. Quest'ultima parola ha dalla sua l'uso manzoniano, nel capitolo XXXI dei Promessi sposi (in cui si parla anche delle "quarantene prescritte" dal tribunale della sanità, non necessariamente di quaranta giorni): "Il terrore della contumacia e dei lazzaretti aguzzava tutti gl'ingegni”, scrive Manzoni.

Dati e riferimenti più dettagliati sulle parole quarantena e contumacia saranno offerti in un articolo di chi scrive e di Alessandro Parenti, che verrà pubblicato prossimamente sulla rivista "Lingua nostra".

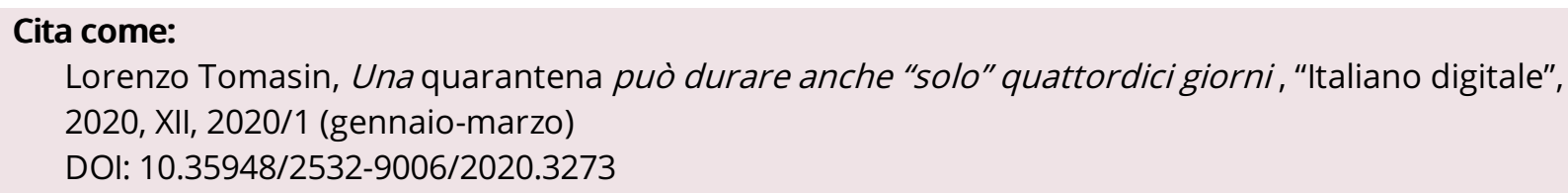

